\title{
Tail of the Spermatozoon
}

National Cancer Institute

\section{Source}

National Cancer Institute. Tail of the Spermatozoon. NCI Thesaurus. Code C33733.

The part of the sperm called the flagellum; it functions to propel the sperm towards the egg. 